\title{
OPTIMAL CONTROL OF A CHEMICAL REACTOR
}

\author{
K. H. WONG and N. LOCK'
}

(Received 21 March 1995; revised 16 November 1995)

\begin{abstract}
A chemical reactor problem is considered governed by partial differential equations. We wish to control the input temperature and the input oxygen concentration so that the actual output temperature can be as close to the desired output temperature as possible. By linearizing the differential equations around a nominal equation and then applying a finiteelement Galerkin Scheme to the resulting system, the original problem can be converted into a sequence of linearly-constrained quadratic programming problems.
\end{abstract}

\section{Introduction}

The simplest model of the tabular chemical reactor can be described by two differential equations (the mass conservation equation and the energy equation) subject to the boundary and initial conditions. The full derivation of these two parabolic equations can be found in [1, 4]. These equations have been solved in [2], although the solutions have not been exploited in full, whereas in [5], the Newton-Raphson iterative algorithm together with collocation methods have been applied to obtain the steady state temperature and concentration profiles for the chemical tabular reaction.

In this paper, a chemical reactor problem is considered similar to those described in $[1,2,4,5]$. The aim is to find suitable control variables so that a desired output temperature is achieved. The variables to be controlled are the temperature and the concentration of oxygen, which are functions of both position and time, and are described by a couple of nonlinear diffusion equations. The control variables are input temperature and input oxygen concentration. The objective function to be minimized is the mean-square error between actual output temperature and the desired output temperature. By linearising the differential equations around a nominal solution and then applying a finite-element Galerkin scheme to the resulting system, the original

\footnotetext{
${ }^{1}$ Dept of Computational and Applied Mathematics, University of the Witwatersrand, Johannesburg, South Africa.

(C) Australian Mathematical Society, 1997, Serial-fee code 0334-2700/97
} 
problem can be converted into a sequence of quadratic programming problems with linear constraints which can be easily solved by any standard quadratic programming software packages. There are several such packages in NAG Library. The above method of solution is strongly motivated by that given in [3].

\section{Nonlinear diffusion equations for the chemical reaction}

From $[1,2,4,5]$, the differential equations governing the chemical reaction are given by

$$
\begin{gathered}
\frac{\partial y}{\partial t}=-f \frac{\partial y}{\partial z}+\frac{1}{P e_{h}} \frac{\partial^{2} y}{\partial z^{2}}+\beta D_{m} R(y, x), \\
0=-f \frac{\partial x}{\partial z}+\frac{1}{P e_{m}} \frac{\partial^{2} x}{\partial z^{2}}-D_{m} R(y, x),
\end{gathered}
$$

where

$$
R(y, x)=x \exp \left\{\gamma\left(1-\left[y\left(1+\alpha T_{r} y\right)\right]^{-1}\right)\right\}
$$

and $x(z, t)$ and $y(z, t)$ are normalized variables representing, respectively, the oxygen concentration $C$ and the temperature $T$ at position $z$ and at time $t$, given by

$$
x(z, t)=C(z, t) / C_{r}
$$

and

$$
y(z, t)=T(z, t) / T_{r}
$$

Here $f$ is a given constant representing the velocity parameter of the reaction; $P e_{h}$, $\beta, D_{m}, P e_{m}, \gamma, \alpha, c_{r}$ and $T_{r}$ are also given constants. Values of these constants taken from a chemistry laboratory are as follows:

$$
\begin{gathered}
f=0.01, \quad P e_{h}=95, \quad \beta=0.38812, \\
D_{m}=0.89173, \quad P e_{m}=235, \quad \gamma=12.297, \quad \alpha=2.5696 \times 10^{-4}, \\
C_{r}=0.01 \text { mole fraction oxygen }(1 \text { mole } \%), \quad T_{r}=434.27 \text { Kelvin. }
\end{gathered}
$$

The boundary conditions for the problem are:

$$
\begin{array}{cc}
\frac{\partial y}{\partial z}\left(z_{f}, t\right)=0, & \frac{\partial x}{\partial z}\left(z_{f}, t\right)=0, \\
y(0, t)=y_{I}(t), & x(0, t)=x_{I}(t), \\
\frac{\partial y}{\partial z}(0, t)=0, & \frac{\partial x}{\partial z}(0, t)=0 .
\end{array}
$$


The variables $x_{I}(t)$ and $y_{I}(t)$, the inlet oxygen concentration and the inlet temperature respectively, are the controls for our problem. Sometimes, the gradient of the inlet oxygen concentration and the gradient of the inlet temperature can also be regarded as controls, in which case equation (8) can be replaced by

$$
\frac{\partial y}{\partial z}(0, t)=\bar{y}_{l}(t), \quad \frac{\partial x}{\partial z}(0, t)=\bar{x}_{l}(t),
$$

where $\bar{y}_{I}(t)$ and $\bar{x}_{I}(t)$ are now additional controls.

Moreover, from the physical point of view, the following constraint needed to be imposed:

$$
x(z, t) \geq 0, \quad z \in\left[0, z_{f}\right], \quad t \in[0, T] .
$$

The objective is to have the outlet temperature $y\left(z_{f}, t\right)$ approach a desired temperature distribution $y_{d}(t)$ as closely as possible. This can be formulated as:

$$
\operatorname{minimize} J=\int_{0}^{1}\left[y\left(z_{f}, t\right)-y_{d}(t)\right]^{2} d t
$$

Here, the time interval $t \in[0,1]$ is used. Any other time interval $t^{\prime} \in[0, T]$ can be converted into the time interval $t \in[0,1]$ by using the transformation $t=t^{\prime} / T$.

\section{Method of solution}

The method of solution used in this section is strongly motivated by that given in Section 6.7 of [3].

A finite-element Galerkin method will be used to convert the distributed optimal control problem into a finite-dimensional mathematical programming problem with the values of the input temperature, the input oxygen concentration, together with their respective gradients at every grid point being the variables. Let the temperature and concentration be approximated as

$$
y(z, t) \approx \sum_{i=1}^{N+1} B_{i}(t) Y_{i}(z)
$$

and

$$
x(z, t) \approx \sum_{i=1}^{N+1} B_{i}(t) X_{i}(z),
$$

where the integer $N$ and the functions $B_{i}(t)$ are yet to be determined. 
Substituting (12) and (13) into (1), we get

$$
\sum_{i=1}^{N+1} \dot{B}_{i}(t) Y_{i}(z)=-f \sum_{i=1}^{N+1} B_{i}(t) Y_{i}^{\prime}(z)+\frac{1}{P e_{h}} \sum_{i=1}^{N+1} B_{i}(t) Y_{i}^{\prime \prime}(z)+\beta D_{m} R(y, x)
$$

Multiplying (14) by $B_{j}(t)$ and integrating over $[0,1]$, we get

$$
\begin{aligned}
\sum_{i=1}^{N+1} Y_{i}(z) & \int_{0}^{1} B_{j}(t) \dot{B}_{i}(t) d t \\
= & -f \sum_{i=1}^{N+1} Y_{i}^{\prime}(z) \int_{0}^{1} B_{j}(t) B_{i}(t) d t+\frac{1}{P e_{h}} \sum_{i=1}^{N+1} Y_{i}^{\prime \prime}(z) \int_{0}^{1} B_{j}(t) B_{i}(t) d t \\
& +\beta D_{m} \int_{0}^{1} R(y, x) B_{j}(t) d t .
\end{aligned}
$$

To convert (15) into a linear differential equation involving $Y_{i}(z)$ and $X_{i}(z)$, the term $R(y, x)$ must be linearised around a suitable steady-state solution $(\bar{y}(z), \bar{x}(z))$ as follows:

$$
R(y, x)=R(\bar{y}, \bar{x})+(y-\bar{y}) R_{y}(\bar{y}, \bar{x})+(x-\bar{x}) R_{x}(\bar{y}, \bar{x}) .
$$

However, from (3)

$$
R(\bar{y}, \bar{x})=\bar{x} R_{x}(\bar{y}, \bar{x}) .
$$

Combining (16) and (17), we get

$$
R(y, x)=(y-\bar{y}) R_{y}(\bar{y}, \bar{x})+x R_{x}(\bar{y}, \bar{x}) .
$$

Whether the above linearization will be acceptable depends entirely upon $\bar{x}$ and $\bar{y}$. One may of course use $\bar{x}$ sufficiently small to give a temperature rise over the reactor which is sufficiently small to render the approximation reasonable. A suitable choice for the steady-state solution is $\bar{y}=0.8175$ and $\bar{x}=0.25$. The reason that these values are chosen is because it has been tested experimentally in the chemistry laboratory that these values work very well for the regulatory control problem. This solution is independent of $z$ and corresponds to a concentration of oxygen of 0.0025 and a temperature of $82^{\circ} \mathrm{C}$. 
In view of (18), (12) and (13), equation (15) can be written as

$$
\begin{aligned}
\sum_{i=1}^{N+1} Y_{i}(z) & \int_{0}^{1} B_{j}(t) \dot{B}_{i}(t) d t \\
= & -f \sum_{i=1}^{N+1} Y_{i}^{\prime}(z) \int_{0}^{1} B_{j}(t) B_{i}(t) d t \\
& +\frac{1}{P e_{h}} \sum_{i=1}^{N+1} Y_{i}^{\prime \prime}(z) \int_{0}^{1} B_{j}(t) B_{i}(t) d t-\beta D_{m} \bar{y} R_{y}(\bar{y}, \bar{x}) \int_{0}^{1} B_{j}(t) d t \\
& +\beta D_{m} R_{y}(\bar{y}, \bar{x}) \sum_{i=1}^{N+1} Y_{i}(z) \int_{0}^{1} B_{j}(t) B_{i}(t) d t \\
& +\beta D_{m} R_{x}(\bar{y}, \bar{x}) \sum_{i=1}^{N+1} X_{i}(z) \int_{0}^{1} B_{j}(t) B_{i}(t) d t .
\end{aligned}
$$

By using exactly the same argument as that used to obtain (19) from (1), we can easily deduce from (2) that

$$
\begin{aligned}
0= & -f \sum_{i=1}^{N+1} X_{i}^{\prime}(z) \int_{0}^{1} B_{j}(t) B_{i}(t) d t+\frac{1}{p e_{m}} \sum_{i=1}^{N+1} X_{i}^{\prime \prime}(z) \int_{0}^{1} B_{j}(t) B_{i}(t) d t \\
& +D_{m} \bar{y} R_{y}(\bar{y}, \bar{x}) \int_{0}^{1} B_{j}(t) d t-D_{m} R_{y}(\bar{y}, \bar{x}) \sum_{i=1}^{N+1} Y_{i}(z) \int_{0}^{1} B_{j}(t) B_{i}(t) d t \\
& -D_{m} R_{x}(\bar{y}, \bar{x}) \sum_{i=1}^{N+1} X_{i}(z) \int_{0}^{1} B_{j}(t) B_{i}(t) d t .
\end{aligned}
$$

Now let

$$
\begin{array}{cc}
X(z)=\left[X_{1}(z), X_{2}(z), \ldots X_{N+1}(z)\right]^{T}, & \bar{X}(z)=X^{\prime}(z) \\
Y(z)=\left[Y_{1}(z), Y_{2}(z), \ldots Y_{N+1}(z)\right]^{T}, & \bar{Y}(z)=Y^{\prime}(z) .
\end{array}
$$

Let the matrices $A$ and $C$ and the vector $b$ be defined as

$$
\begin{aligned}
A_{j i} & =\int_{0}^{1} B_{j}(t) B_{i}(t) d t, \quad i, j=1, \ldots, N+1, \\
C_{j i} & =\int_{0}^{1} B_{j}(t) \dot{B}_{i}(t) d t, \quad i, j=1, \ldots, N+1, \\
b_{i} & =\int_{0}^{1} B_{j}(t) d t .
\end{aligned}
$$

By using a suitable choice of $B_{i}(t)$, such as the one defined by equation (32) later, the matrix $A$ in equation (23) can be made invertible. 
Then the equations (19) and (20) can be written as

$$
\begin{aligned}
X^{\prime}(z)= & \bar{X}(z) \\
\bar{X}^{\prime}(z)= & f P e_{m} \bar{X}(z)+P e_{m} D_{m} R_{y}(\bar{y}, \bar{x}) Y(z) \\
& +P e_{m} D_{m} R_{x}(\bar{y}, \bar{x}) X(z)-P e_{m} D_{m} \bar{y} R_{y}(\bar{y}, \bar{x}) A^{-1} b \\
Y^{\prime}(z)= & \bar{Y}(z) \\
\bar{Y}^{\prime}(z)= & f P e_{h} \bar{Y}(z)+\left(P e_{h} A^{-1} C-\beta D e_{h} D_{m} R_{y}(\bar{y}, \bar{x}) I_{N+1}\right) Y(z) \\
& -P e_{h} \beta D_{m} R_{x}(\bar{y}, \bar{x}) X(z)+P e_{h} \beta D_{m} \bar{y} R_{y}(\bar{y}, \bar{x}) A^{-1} b .
\end{aligned}
$$

Let

$$
\chi(z)=\left[X^{T}(z), \bar{X}^{T}(z), Y^{T}(z), \bar{Y}^{T}(z)\right]^{T} .
$$

Then

$$
\chi^{\prime}(z)=\Gamma \chi(z)+\hat{\Gamma}
$$

where

$$
\begin{gathered}
\Gamma=\left[\begin{array}{cccc}
0 & I_{N+1} & 0 & 0 \\
K_{1} I_{N+1} & K_{2} I_{N+1} & K_{3} I_{N+1} & 0 \\
0 & 0 & 0 & I_{N+1} \\
K_{4} I_{N+1} & 0 & \hat{A} & K_{5} I_{N+1}
\end{array}\right] \\
\hat{\Gamma}=\left[\begin{array}{c}
0 \\
K_{6} \\
0 \\
K_{7}
\end{array}\right]
\end{gathered}
$$

and

$$
\begin{gathered}
K_{1}=P e_{m} D_{m} R_{x}(\bar{y}, \bar{x}), \quad K_{2}=f P e_{m}, \quad K_{3}=P e_{m} D_{m} R_{y}(\bar{y}, \bar{x}), \\
K_{4}=-P e_{h} \beta D_{m} R_{x}(\bar{y}, \bar{x}), \quad K_{5}=f P e_{h}, \quad K_{6}=-P e_{m} D_{m} \bar{y} R_{y}(\bar{y}, \bar{x}) A^{-1} b, \\
K_{7}=P e_{h} \beta D_{m} \bar{y} R_{y}(\bar{y}, \bar{x}) A^{-1} b \\
\hat{A}=P e_{h} A^{-1} C-\beta P e_{h} D_{m} R_{y}(\bar{y}, \bar{x}) I_{N+1} .
\end{gathered}
$$

So far, the functions $B_{i}(t)$ have not been specified. A suitable choice of $B_{i}(t)$ is the linear $B$ splines function defined on the time interval $[0,1]$ with $N$ equal subintervals. That is, let

$$
B_{i}(t)= \begin{cases}2+N t-i, & t \in[(i-2) / N,(i-1) / N] \\ i-N t, & t \in[(i-1) / N, i / N] \\ 0, & \text { otherwise. }\end{cases}
$$


Then the elements of $A, C$ and $b$ are given by

$$
\begin{gathered}
A_{1,1}=A_{N+1, N+1}=\frac{1}{3 N}, \quad A_{i, i}=\frac{2}{3 N}, \quad i=2, \ldots, N \\
A_{i, i+1}=\frac{1}{6 N}, \quad i=1 \ldots N, \quad A_{i, i-1}=\frac{1}{6 N}, \quad i=2 \ldots N+1
\end{gathered}
$$

and $A_{i, j}=0$ otherwise;

$$
\begin{array}{cl}
C_{1,1}=-\frac{1}{2}, & C_{N+1, N+1}=\frac{1}{2}, \\
C_{i, i+1}=\frac{1}{2}, \quad i=1,2 \ldots N, & C_{i, i-1}=-\frac{1}{2}, \quad i=2 \ldots N+1
\end{array}
$$

and $C_{i, j}=0$ otherwise;

$$
b_{1}=b_{N+1}=\frac{1}{2 N}, \quad b_{i}=\frac{1}{N}, \quad i=2, \ldots . N
$$

From (30), it is clear that

$$
\begin{aligned}
\chi(z) & =e^{\Gamma z} \chi(0)+\int_{0}^{z} e^{\Gamma(z-t)} \hat{\Gamma} d t \\
& =e^{\Gamma z} \chi(0)+r(z)
\end{aligned}
$$

where

$$
r(z)=\left(e^{\Gamma z}-I_{4 N+4}\right) \Gamma^{-1} \hat{\Gamma} .
$$

In view of (12), (23) and (33), the objective function (11) can be written as

$$
\begin{aligned}
& \operatorname{minimize} J= \int_{0}^{1}\left[\sum_{i=1}^{N+1} B_{i}(t) Y_{i}\left(Z_{f}\right)-y_{d}(t)\right]^{2} d t \\
&=\int_{0}^{1}\left[\sum_{i=1}^{N+1} B_{i}^{2}(t) Y_{i}^{2}\left(Z_{f}\right)+2 \sum_{i=1}^{N} B_{i}(t) B_{i+1}(t) Y_{i}\left(Z_{f}\right) Y_{i+1}\left(Z_{f}\right)\right. \\
&\left.\quad-2 y_{d}(t) \sum_{i=1}^{N+1} B_{i}(t) Y_{i}\left(Z_{f}\right)+y_{d}^{2}(t)\right] d t \\
&=y\left(z_{f}\right)^{T} \hat{H} Y\left(z_{f}\right)+\hat{g}^{T} Y\left(z_{f}\right)+\hat{C},
\end{aligned}
$$


where the matrix $\hat{H}$, the vector $\hat{g}$ and the real number $\hat{C}$ are given by

$$
\begin{gathered}
\hat{H}_{1,1}=\hat{H}_{N+1, N+1}=\frac{1}{3 N}, \quad \hat{H}_{i i}=\frac{2}{3 N}, \quad i=2, \ldots, N, \\
\hat{H}_{i, i+1}=\frac{1}{6 N}, \quad i=1, \ldots, N, \quad \hat{H}_{i, i-1}=\frac{1}{6 N}, \quad i=2, \ldots, N+1, \\
\hat{H}_{i j}=0, \quad \text { otherwise } \\
\hat{g}_{i}=\int_{0}^{1}-2 y_{d}(t) B_{i}(t) d t, \quad i=1, \ldots, N+1
\end{gathered}
$$

and

$$
\hat{C}=\int_{0}^{1} y_{d}^{2}(t) d t
$$

Now, since the functions $B_{i}(t)$ are known, $\hat{g}$ and $\hat{C}$ can be easily computed for any given function $y_{d}(t)$. In particular, when $y_{d}(t)=y_{d}$ is a constant, then

$$
\begin{gathered}
\hat{g}_{1}=\hat{g}_{N+1}=\frac{y_{d}}{N} \\
\hat{g}_{i}=\frac{2 y_{d}}{N}, \quad i=2, \ldots, N .
\end{gathered}
$$

In view of (30), the objective function (37) can be written as

$$
\operatorname{minimize} J=\chi^{T}\left(z_{f}\right) H^{*} \chi\left(z_{f}\right)+g^{*^{T}} \chi\left(z_{f}\right)+\hat{k}
$$

where

$$
\begin{gathered}
H^{*}=\left[\begin{array}{llll}
0 & 0 & 0 & 0 \\
0 & 0 & 0 & 0 \\
0 & 0 & \hat{H} & 0 \\
0 & 0 & 0 & 0
\end{array}\right], \\
g^{*}=\left[\begin{array}{l}
\mathbf{0} \\
\mathbf{0} \\
\hat{g} \\
\mathbf{0}
\end{array}\right],
\end{gathered}
$$

0 is the $N+1$ zero matrix and 0 is the $N+1$ zero vector.

We can substitute (36a) into (39) to get

$$
\operatorname{minimize} J=\chi^{T}(0) \hat{H} \chi(0)+\hat{g}^{T} \chi(0)+\hat{k},
$$


where

$$
\begin{aligned}
\hat{H} & =\left(e^{\Gamma z_{f}}\right)^{T} H^{*} e^{\Gamma z_{f}}, \\
\hat{g} & =\left(e^{\Gamma z_{f}}\right)^{T}\left[\left(H+H^{*^{T}}\right) r\left(z_{f}\right)+g^{*}\right], \\
\hat{k} & =r^{T}\left(z_{f}\right) H^{*} r\left(z_{f}\right)+\left(g^{*}\right)^{T} r\left(z_{f}\right)+\hat{C} .
\end{aligned}
$$

Now, in view of (12), (13), (21), (22), (30), the boundary condition (4) can be written as

$$
\begin{aligned}
& \sum_{i=1}^{N+1} B_{i}(t) Y_{i}^{\prime}\left(z_{f}\right)=\sum_{i=1}^{N+1} B_{i}(t) X_{i}^{\prime}\left(z_{f}\right)=0 \\
\Rightarrow & \sum_{i=1}^{N+1} B_{i}(t) \bar{Y}_{i}\left(z_{f}\right)=\sum_{i=1}^{N+1} B_{i}(t) \bar{X}_{i}\left(z_{f}\right)=0 \\
\Rightarrow & \sum_{i=1}^{N+1} B_{i}(t) \chi_{3 N+3+i}\left(z_{f}\right)=\sum_{i=1}^{N+1} B_{i}(t) \chi_{N+1+i}\left(z_{f}\right)=0
\end{aligned}
$$

By letting $t$ to be equal to the grid points $0,1 / N, 2 / N, \ldots 1$, we easily obtain from (49) and (36a) that

$$
\begin{gathered}
\left\{e^{\Gamma z_{f}} \chi(0)+r\left(z_{f}\right)\right\}_{i}=0 \\
i=N+2, \ldots, 2 N+2 \text { and } 3 N+4, \ldots, 4 N+4 .
\end{gathered}
$$

Similarly, the boundary condition (6) can be written as

$$
\begin{gathered}
\bar{Y}(0)=\bar{X}=0 \Rightarrow \chi_{i}(0)=0 \\
i=N+2, \ldots, 2 N+2 \text { and } 3 N+4, \ldots, 4 N+4
\end{gathered}
$$

Again by letting $t$ to be equal to the grid points $0,1 / N, 2 / N, \ldots 1$, we obtain from (10), (13) and (30) that

$$
\chi_{i}(z) \geq 0, \quad i=1, \ldots, N+1
$$

Finally, we shall approximate the above constraint by the constraint

$$
\chi_{i}\left(z^{j}\right) \geq 0, \quad i=1, \ldots, N+1 ; \quad j=1, \ldots, S,
$$

where the points $z^{j}$ are chosen such that

$$
0=z^{1}<z^{2}<\cdots<z^{S}=z_{f} .
$$

In view of (36), the constraint (53) becomes

$$
\left\{e^{\Gamma z^{j}} \chi(0)+r\left(z^{j}\right)\right\}_{i} \geq 0, \quad i=1, \ldots, N+1 ; j=1, \ldots, S .
$$


Now, let

$$
u \equiv \chi(0)
$$

Then, in view of (44), (49), (50) and (54), the problem with the gradient of the inlet temperature and the gradient of the inlet oxygen concentration equal to zero can be written as

$$
(\mathrm{P} 1)\left\{\begin{array}{l}
\operatorname{minimize}_{u \in R^{4 N+4}} J=\frac{1}{2} u^{T} \hat{H} u+\hat{g}^{T} u+\hat{k} \\
\text { subject to } \\
\left\{e^{\Gamma z_{f}} u\right\}_{i}=-\Gamma_{i}\left(z_{f}\right) \\
i=N+2, \ldots, 2 N+2 \text { and } 3 N+4, \ldots, 4 N+4, \\
u_{i}=0, \\
i=N+2, \ldots, 2 N+2 \quad \text { and } 3 N+2, \ldots, 4 N+4 \\
\text { and } \\
\left\{e^{\Gamma z^{j}} \chi(0)\right\}_{i} \geq-r_{i}\left(z^{j}\right), \quad i=1, \cdots, N+1 ; \quad j=1, \ldots, S .
\end{array}\right.
$$

Similarly, the problem with the gradient of the inlet temperature and the gradient of the inlet oxygen concentration being regarded as additional controls can be written as

$$
(\mathrm{P} 2)\left\{\begin{array}{l}
\operatorname{minimize}_{u \in R^{4 N+4}} \quad J=\frac{1}{2} u^{T} \hat{H} u+\hat{g}^{T} u+\hat{k} \\
\text { subject to } \left.^{\Gamma z_{f}} u\right\}_{i}=-\Gamma_{i}\left(z_{f}\right), \\
i=N+2, \ldots, 2 N+2 \text { and } 3 N+4, \ldots, 4 N+N \\
\text { and } \\
\left\{e^{\Gamma z^{j}} \chi(0)\right\}_{i} \geq-r_{i}\left(z^{j}\right), \quad i=1, \cdots, N+1 ; \quad j=1, \cdots, S .
\end{array}\right.
$$

Both $(P 1)$ and $(P 2)$ are quadratic programming problems with linear constraints, which can be easily solved by any standard quadratic programming software.

Once the optimal value of $u$ is found, the optimal control can be easily found by the formulae

$$
\begin{aligned}
& x_{l}(t)=x(0, t)=\sum_{i=1}^{N+1} B_{i}(t) X_{i}(0)=\sum_{i=1}^{N+1} B_{i}(t) u_{i} \\
& y_{I}(t)=y(0, t)=\sum_{i=1}^{N+1} B_{i}(t) Y_{i}(0)=\sum_{i=1}^{N+1} B_{i}(t) u_{2 N+2+i}(0) \\
& \bar{x}_{I}(t)=\frac{\partial x}{\partial z}(0, t)=\sum_{i=1}^{N+1} B_{i}(t) X_{i}^{\prime}(0)=\sum_{i=1}^{N+1} B_{i}(t) u_{N+1+i} \\
& \bar{y}_{I}(t)=\frac{\partial y}{\partial z}(0, t)=\sum_{i=1}^{N+1} B_{i}(t) Y_{i}^{\prime}(0)=\sum_{i=1}^{N+1} B_{i}(t) u_{3 N+3+i}
\end{aligned}
$$


The temperature and concentration and their respective gradient at any time $t$ and at any position $z$ can be easily obtained by the formulae

$$
\begin{aligned}
x(z, t) & =\sum_{i=1}^{N+1} B_{i}(t) \chi_{i}(z), \\
y(z, t) & =\sum_{i=1}^{N+1} B_{i}(t) \chi_{2 N+2+i}, \\
x^{\prime}(z, t) & =\sum_{i=1}^{N+1} B_{i}(t) \chi_{N+1+i}(z), \\
y^{\prime}(z, t) & =\sum_{i=1}^{N+1} B_{i}(t) \chi_{2 N+2+i}(z),
\end{aligned}
$$

where $\chi(z)$ can be found from (36) and (55).

\section{Computational aspects and implementation}

Only two aspects of implementing the above method need discussion. They are

(i) the evaluation of $\Gamma(z)$

(ii) the calculation of the exponential matrix $e^{\Gamma z}$.

From (36b), the evaluation of $r(z)$ involves the calculation of the inverse of $\Gamma^{-1}$. However, $\Gamma$ is extremely ill-conditioned since the reciprocal of the condition number of $\Gamma$ is of order $10^{-18}$. Thus, any attempt to calculate the inverse of $\Gamma$ will fail numerically. Hence, $r(z)$ cannot be calculated directly from (36b). Instead, we use the Gaussian quadrature rule of order 5 to evaluate the integral

$$
r(z)=\int_{0}^{z} e^{\Gamma(z-t)} \hat{\Gamma} d t
$$

The ill-conditioning method of $\Gamma$ also affected the choice of method to calculate the exponential matrix $e^{\Gamma z}$. No numerical method is known for calculating the eigenvalues of $\Gamma$. Thus, we need to find some methods which does not involve the eigenvalues of $\Gamma$. Two approaches can be adopted.

Firstly, we consider the series expansion of the exponential matrix, viz.

$$
e^{\Gamma z}=\sum_{i=0}^{\infty} \frac{\Gamma^{i} z^{i}}{i !}
$$

The series is considered to have converged when the difference between two successive intermediate matrices is less than $10^{-10}$ in both the $L_{1}$ and $L_{\infty}$ matrix 
norm. These norms are calculated by the IMSL routines DNRIRR and DNRIRR respectively. However, the series-expansion method to calculate the exponential matrix can sometimes create two types of errors, namely, roundoff and cancellation errors, which can render the method unusable. Thus, a second method is used in order to check the accuracy of the result that we obtain in the series-expansion method. This method involves scaling and the Padé approximation. It has been found that the rate of convergence of the second method was faster than that of the first method, but there was no significant difference between the results produced by the two methods.

A further difficulty in calculating the exponential matrix is encounted in the choice of $z_{f}$. For $z_{f}>0.1$, the exponential matrix consists of some element which is so large that they cannot be handled by the computer. As a compromise, we choose $z_{f}$ to be 0.1 .

Finally, the IMSL routine DQPROG, based on an algorithm by Goldfarb, is used to solve the quadratic programming problem (P1) and (P2).

\section{Illustrative examples}

EXAMPLE 5.1. We consider the problem (P2) with the following desired outlet temperature $y_{d}=0.7,0.63,0.5$ and 0.8 , which corresponds to $31^{\circ} \mathrm{C}, 0^{\circ} \mathrm{C},-55^{\circ} \mathrm{C}$ and $74^{0} \mathrm{C}$ respectively.

For $y_{d}=0.7$, the problem has been solved with $N=4,8$ and 12. For $y_{d}=0.63$ and 0.5 , the problem has been solved with $N=8$. For $y_{d}=0.8$, the problem has been solved with $N=4$ only.

In all cases, the optimal solutions have the following properties.

(i) The optimal value of $J$ is extremely closed to zero, which shows that the actual output temperature is almost the same as the desired output temperature.

(ii) The boundary condition (6) is satisfied with the value of $x^{\prime}\left(z_{f}, t\right)$ and $y^{\prime}\left(z_{f}, t\right)$ being of order $10^{-14}$ or less.

(iii) The controls $x(0, t)$ and $y(0, t)$ are almost constant functions of $t$, with the input temperature $y(0, t)$ almost the same as the desired output temperature for all $t \in[0,1]$. The variations of the controls $x^{\prime}(0, t)$ and $y^{\prime}(0, t)$ are also very small as $t$ varies from 0 to 1 .

(iv) For each fixed $z$, the oxygen concentration $x(z, t)$ and the temperature $y(z, t)$ remain almost constant with time.

(v) For each fixed $t$, the oxygen concentration $x(z, t)$ increases along the reactor, while the temperature $y(z, t)$ decreases along the vector.

The numerical results are summarized in Table 1-4. Graphs of the optimal controls for $y_{d}=0.7$ and $N=12$ are plotted in Figure 1. 
TABLE 1. Outlet temperature $=0.7$. No. of partitions used $=12$.

\section{The optimal controls are:}

$\begin{array}{ccccc}t & x(0, t) & x^{\prime}(0, t) & y(0, t) & y^{\prime}(0, t) \\ 0.000 & 0.498 & 0.134 & 0.701 & -0.022 \\ 0.167 & 0.498 & 0.135 & 0.701 & -0.023 \\ 0.333 & 0.503 & 0.119 & 0.701 & -0.021 \\ 0.500 & 0.501 & 0.128 & 0.701 & -0.016 \\ 0.667 & 0.502 & 0.122 & 0.701 & -0.023 \\ 0.833 & 0.502 & 0.124 & 0.701 & -0.022 \\ 1.000 & 0.492 & 0.156 & 0.701 & -0.039\end{array}$

The boundary condition of the problem

$\begin{array}{ccc}t & x^{\prime}(0.1, t) & y^{\prime}(0.1, t) \\ 0.000 & -9.2 \times 10^{-14} & 4.4 \times 10^{-15} \\ 0.167 & -8.5 \times 10^{-14} & 0.0 \\ 0.333 & -8.9 \times 10^{-14} & -2.2 \times 10^{-15} \\ 0.500 & -7.8 \times 10^{-14} & 5.5 \times 10^{-15} \\ 0.667 & -8.2 \times 10^{-14} & 6.1 \times 10^{-15} \\ 0.833 & -8.9 \times 10^{-14} & 1.7 \times 10^{-15} \\ 1.000 & -7.8 \times 10^{-14} & 6.7 \times 10^{-15}\end{array}$

TABLE 2. Outlet temperature $=0.63$. No. of partitions $=8$.

\section{The optimal controls are:}

$\begin{array}{ccccc}t & x(0, t) & x^{\prime}(0, t) & y(0, t) & y^{\prime}(0, t) \\ 0.000 & 0.794 & 0.220 & 0.632 & -0.035 \\ 0.250 & 0.794 & 0.219 & 0.632 & -0.038 \\ 0.500 & 0.794 & 0.220 & 0.632 & -0.037 \\ 0.750 & 0.795 & 0.220 & 0.632 & -0.036 \\ 1.000 & 0.798 & 0.206 & 0.632 & -0.028\end{array}$

The boundary condition of the problem

$\begin{array}{ccc}t & x^{\prime}(0.1, t) & y^{\prime}(0.1, t) \\ 0.000 & -4.3 \times 10^{-14} & 8.9 \times 10^{-16} \\ 0.250 & -5.0 \times 10^{-14} & 4.9 \times 10^{-15} \\ 0.500 & -5.3 \times 10^{-14} & -2.9 \times 10^{-15} \\ 0.750 & -5.0 \times 10^{-14} & -2.2 \times 10^{-15} \\ 1.000 & -4.6 \times 10^{-14} & 1.2 \times 10^{-14}\end{array}$


TABLE 3. Outlet temperature $=0.5$. No. of partitions used $=8$.

The optimal controls are:

$\begin{array}{ccccc}t & x(0, t) & x^{\prime}(0, t) & y(0, t) & y^{\prime}(0, t) \\ 0.000 & 1.344 & 0.373 & 0.503 & -0.062 \\ 0.250 & 1.345 & 0.369 & 0.503 & -0.063 \\ 0.500 & 1.345 & 0.371 & 0.503 & -0.063 \\ 0.750 & 1.348 & 0.362 & 0.503 & -0.063 \\ 1.000 & 1.351 & 0.352 & 0.503 & -0.046\end{array}$

The boundary conditions of the problem

$\begin{array}{ccc}t & x^{\prime}(0.1, t) & y^{\prime}(0.1, t) \\ 0.000 & -3.9 \times 10^{-14} & 2.7 \times 10^{-15} \\ 0.250 & -3.6 \times 10^{-14} & 8.9 \times 10^{-15} \\ 0.500 & -4.0 \times 10^{-14} & 2.2 \times 10^{-15} \\ 0.750 & -3.6 \times 10^{-14} & 6.9 \times 10^{-15} \\ 1.000 & -3.6 \times 10^{-14} & 7.1 \times 10^{-15}\end{array}$

TABLE 4. Outlet temperature $=0.8$. No. of partitions used $=4$.

The optimal controls are:

$\begin{array}{ccccc}t & x(0, t) & x^{\prime}(0, t) & y(0, t) & y^{\prime}(0, t) \\ 0.000 & 0.000 & 0.282 & 0.802 & -0.047 \\ 0.250 & 0.000 & 0.282 & 0.802 & -0.047 \\ 0.500 & 0.000 & 0.282 & 0.802 & -0.047 \\ 0.750 & 0.000 & 0.282 & 0.802 & -0.047 \\ 1.000 & 0.000 & 0.282 & 0.802 & -0.047\end{array}$

The boundary conditions of the problem:

$\begin{array}{ccc}t & x^{\prime}(0.1, t) & y^{\prime}(0.1, t) \\ 0.000 & -2.5 \times 10^{-14} & -8.9 \times 10^{-16} \\ 0.250 & -2.5 \times 10^{-14} & 4.2 \times 10^{-15} \\ 0.500 & -2.5 \times 10^{-14} & 0.000 \\ 0.750 & -1.8 \times 10^{-14} & 3.1 \times 10^{-15} \\ 1.000 & -1.8 \times 10^{-14} & 5.6 \times 10^{-15}\end{array}$




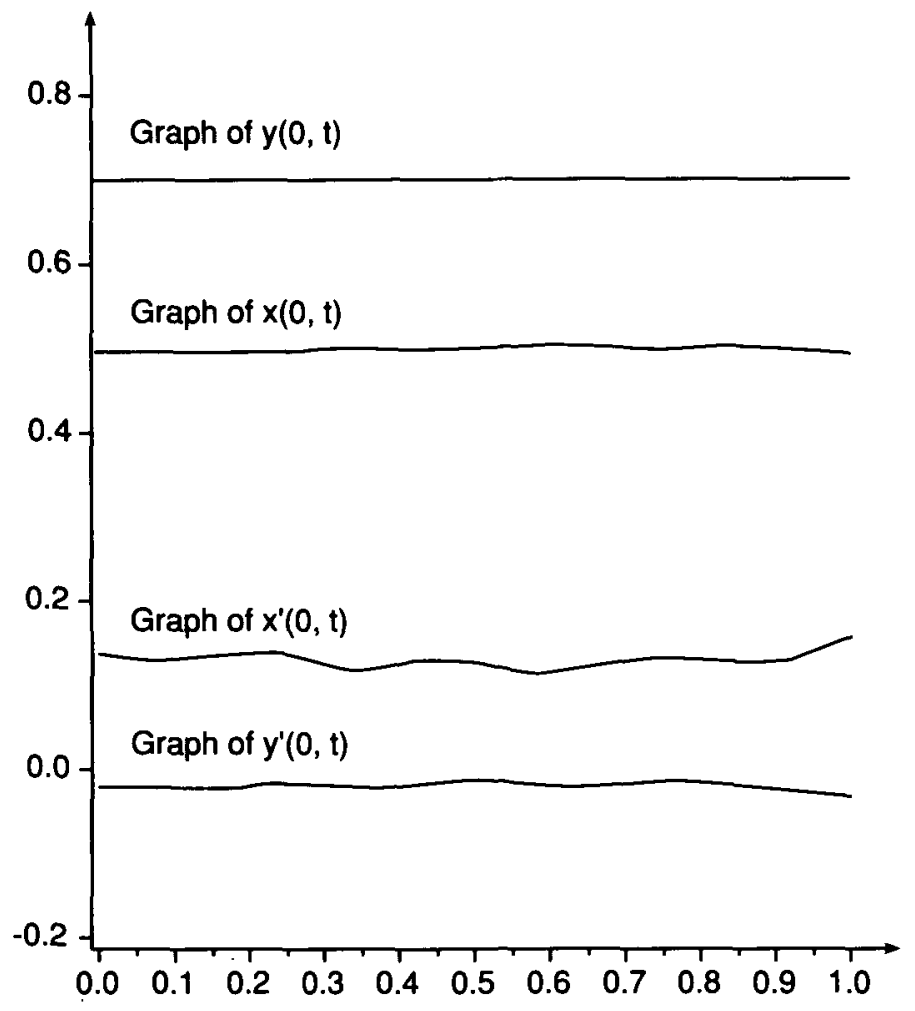

FIGURE 1. Optimal controls $y_{d}=0.7, N=12$.

\section{Conclusion}

From the numerical results obtained in Section 5, it appears that the method described in this paper works very well for the problem (P2) described in Section 3. In all the numerical examples solved, the actual output temperature obtained is almost the same as the desired output temperature. In general, an increase in the number of partitions $N$ over the time interval $[0,1]$ will lead to the convergence of the optimal control of the approximate problems to the true optimal control.

\section{References}

[1] N. R. Amundson, "Mathematical models for fixed bed reactors", Berichte der Bunsen Gesellschaft 74 (1970) 90-98.

[2] S. L. Liu and N. R. Amundson, "Stability of adiabatic packed-bed reactors, effect of axial mixing", Ind. \& Eng. Chem. Fund. 2 (1963) 183. 
[3] K. L. Teo and Z. S. Wu, Computational Methods for Optimizing Distributed Systems (Academic Press, Orlando, 1984).

[4] A. Varma and R. Aris, Stirred pots and empty tubes (Prentice-Hall, Englewood Cliffs, N. J., 1976).

[5] A. Varma, C. Georgakis, N. R. Amundson and R. Aris, "Computational methods for the tabular chemical reactor", Comp. Methods in Appl. Mech. and Eng. 8 (1976) 319. 